\title{
Multilinguales
}

\section{Alternance lexicale : structures, situations et domaines d'emploi}

Lexical alternation: structures, situations and fields of employment

\section{Moufoutaou Adjeran}

\section{(2) OpenEdition}

\section{Journals}

\section{Édition électronique}

URL : https://journals.openedition.org/multilinguales/4814

DOI : $10.4000 /$ multilinguales.4814

ISSN : 2335-1853

\section{Éditeur}

Université Abderrahmane Mira - Bejaia

\section{Référence électronique}

Moufoutaou Adjeran, «Alternance lexicale : structures, situations et domaines d'emploi », Multilinguales [En ligne], 13 | 2020, mis en ligne le 15 juillet 2020, consulté le 18 mai 2021. URL : http:// journals.openedition.org/multilinguales/4814; DOI : https://doi.org/10.4000/multilinguales.4814

Ce document a été généré automatiquement le 18 mai 2021

\section{(c) $(1)(9)$}

Multilinguales est mise à disposition selon les termes de la Licence Creative Commons Attribution Pas d'Utilisation Commerciale - Pas de Modification 4.0 International 


\section{Alternance lexicale : structures, situations et domaines d'emploi}

Lexical alternation: structures, situations and fields of employment

Moufoutaou Adjeran

1 Le concept d'alternance codique (Fishman, 1971 et 1972 ; Gumperz et al, 1972 ; MyersScotton, 1993) a constitué l'un des fers de lance de la sociolinguistique de contact des langues. S'il est vrai qu'il a permis des progrès notamment en matière de connaissance des situations (socio)linguistiques caractéristiques de contact des langues et de productions langagières dans les pays colonisés, il apparaît aussi qu'il n'est pas descriptif de toutes les dynamiques (socio)linguistiques observables dans ces milieux. La pratique du français et / ou de l'anglais dans le cadre d'étude défini, laisse apprécier des alternances lexicales confondues, jusque-là, à la néologie (Guilbert, 1973; Aubert, 1979 ; Lüdi, 1980 et 1983 ; Merle et al. 1987, 1992 et 1994 ; Sablayrolles, 1997 ; Bague, 1998 ;Anokhina, 2003 ;Pooe, 2008). L'alternance lexicale est un procédé d'enrichissement en situation de contact des langues (Adjeran, Ndao et Diouf, 2018) et s'opère au niveau lexical. Elle consiste en la création de nouveaux lexèmes dont la base relève étymologiquement d'une Langue Nationale (LN) ou d'une deuxième langue de colonisation et l'affixe adjoint provient toujours du français (F) rarement de l'anglais (A). Le choix du contexte colonial africain ne signifie pas que nos méthodes ne soient pas transférables à d'autres situations de contacts des langues dans lesquelles n'entreraient pas le français ou l'anglais; nous pensons au contraire que le modèle proposé ici a une validité et une portée universelle et peut servir à appréhender le multilinguisme des pays. C'est ce que confirme justement Pooe $(2008: 318)$ quand il avance :

Reflétant l'hétérogénéité du contexte linguistique des townships de Soweto, les particularités lexicales hybrides sont de diverses origines. Cependant, leur principale caractéristique commune c'est que ce sont des combinaisons des lexèmes issus principalement de l'anglais et de l'afrikaans et des affixes des langues bantoues (sotho ou zoulou). Tous les verbes provenant de l'anglais ou de l'afrikaans sont greffés d'un affixe sotho ou zoulou. Le verbe 'buy' (acheter en anglais) devient baya en scamto : on ajoute le suffixe -a au lexème anglais respectant donc la forme des verbes sotho. Au passé on aura bayile, ajoutant donc le suffixe -ile du passé. 
Pour les noms, le pluriel est souvent formé par l'ajout d'un préfixe (ama-/ma-/dietc.) comme dans les noms bantous. Par exemple, 'demons' (anglais pour démons) devient madimon. Les hybrides sont des indicateurs de la nouvelle identité véhiculée par le scamto, principalement celle de la diversité culturelle: ils témoignent d'une situation sociolinguistique de plurilinguisme, et d'une hétérogénéité linguistique qui entraîne des mélanges de langues de différentes familles, fonctionnant alors comme des indices d'homogénéisation, ce qui reflète la diversité des townships et du pays en général, qui s'harmonise progressivement en une seule identité sud-africaine.Pooe (2008 : p. 318).

2 L'objectif de cet article est d'analyser la structure, les situations et les domaines d'emploi des alternances lexicales à partir de la situation linguistique de quelques exterritoires d'expansion et de colonisation (Bénin, Togo, Burkina-Faso, Côte-d'Ivoire, Sénégal, l'Algérie et le Cameroun). Nous faisons l'hypothèse que l'alternance lexicale est constitutive des situations de contact des langues et met en évidence, dans le cas convoqué, l'expression de l'héritage colonial et traduit une cohabitation harmonieuse entre les langues en contact empreinte de la permanente présence du français et ou de l'anglais. Quels sont les situations et les domaines constitutifs du phénomène d'alternance lexicale? Quels en sont les procédés typiques qui résultent de leurs structures? Quel contexte linguistique valide leur pratique? Telles sont les interrogations qui fondent notre problématique. Notre objet d'étude sera organisé selon une structuration en cinq points: dans un premier temps, nous précisons la démarche méthodologique qui fonde ce travail. Dans un deuxième temps, nous établissons une distinction entre néologie et alternance lexicale; dans un troisième temps, nous présentons les recherches sur les alternances codiques afin de voir si l'alternance lexicale n'en n'est pas une manifestation subséquente; dans un quatrième temps, nous analysons les structures des alternances lexicales; dans un cinquième temps, nous traitons des situations et des domaines d'emploi des alternances lexicales.

\section{Démarche méthodologique}

3 Trois approches fondent notre démarche méthodologique : l'observation, l'entretien et des matériaux non enregistrés. Nous avons, d'une part, procédé par observation in situ (Béal, 2000) des locuteurs et par entretien; d'autre part, nous avons consulté la littérature, notamment les journaux africains (cf. références sitographiques) sur supports papier et numérique. Nous avons utilisé des matériaux non enregistrés représentatifs soit d'un type oral formel lorsqu'il proviennent, par exemple de l'écoute d'émissions radiophoniques ou télévisées, des discours, des conférences, des communications, d'interventions dans des représentations théatrales, des débats ou des assemblées soit d'un oral informel: conversations familières entre collègues de service, en famille ou entre amis, même des énoncés saisis au vol dans les rues, les transports, les restaurants, les marchés et autres.

4 Un des objectifs du recueil des données est de privilégier la diversité des contextes d'interactions et la confrontation entre les différentes données. L'entretien ne constitue qu'un mode d'interaction parmi d'autres possibles. Indépendamment du problème de l'authenticité des données, il semble alors singulièrement réducteur de se limiter à cette seule source de données. À la suite de Beaud (1995), nous faisons le choix de l'intégration des entretiens et des observations dans l'analyse. 
5 Nous insistons sur ces acquis méthodologiques concernant l'articulation entre les contextes immédiats et les domaines d'usage des alternances lexicales. Cette double contextualisation interroge la notion d'authenticité et implique dans le même temps de confronter les entretiens aux observations. Ces considérations permettront de situer notre réflexion sur les relations entre les situations et les domaines d'emploi des alternances convoquées et de définir le champ de réflexion dans lequel elle s'insère. La consultation de la littérature (journaux et œuvres littéraires) se justifie par la volonté d'apprécier le degré de formalisation des alternances lexicales. Les matériaux de type oral formel et d'un oral informel laissent appréhender la diversité des contextes d'usages des alternances convoquées et laissent sous-entendre que des personnes dans un contexte et dans un domaine précis offrent des possibilités de création lexicale. Il devient de fait intéressant de s'interroger sur les différentes manières de motiver le signe linguistique pour en apprécier la portée sociolinguistique et pragmatique. La méthodologie ainsi déclinée facilite une approche holistique des alternances lexicales qu'il importe de distinguer de la néologie.

\section{Distinction néologie et alternance lexicale?}

6 Notre objectif n'est pas de procéder à l'inventaire des caractéristiques des néologismes - les travaux pionniers de Guilbert (1975), de Sablayrolles (2000) et de tant d'autres comportent des descriptions très fines de la néologie - nous voudrions simplement voir en quoi elle peut être différente de l'alternance lexicale en vue de construire une lecture précise des deux mécanismes.

7 La néologie s'avère un domaine qui pose beaucoup de problèmes concernant généralement les aspects suivants : la définition des principaux concepts opérationnels, la forme, la fréquence, l'origine et le sémantisme des mots considérés comme appartenant à la classe des néologismes. En plus, parmi les autres domaines de la linguistique, elle s'individualise par sa particularité de désigner, à la fois, ses opérations (les procédés de création) et ses résultats (les néologismes).

8 Par conséquent, la dimension polysémique du terme de néologie est plus qu'évidente car le concept qu'il désigne renvoie à trois démarches différentes :

création de nouvelles unités lexicales par le recours, conscient ou inconscient, aux mécanismes habituels de créativité linguistique d'une langue ;

étude théorique et appliquée des procédés de formation des mots, des critères de reconnaissance, d'acceptabilité et de diffusion des néologismes ;

activité institutionnelle organisée qui se propose de recenser, de créer, de diffuser et d'implanter les néologismes dans le cadre d'une politique linguistique.

9 Partant de ces précisions, nous faisons la remarque selon laquelle la néologie n'implique pas une situation particulière de l'environnement linguistique. Elle s'opère à l'intérieur d'une même langue dans la même logique des procédés habituels de création des unités lexicales. C'est justement ce que précise Guilbert (1973: p.16) lorsqu'il avance :

[...] Toutes les formations lexicales ne relèvent pas du même type de création. Elles se répartissent, pour nous en tenir momentanément à une classification imparfaite, entre créations morphologiques et créations sémantiques. Dans la première forme, ce qui est acte individuel de création, c'est la jonction de deux éléments préexistants, base et affixe, pour engendrer un nouveau mot $($ radar + iste $=$ 
radariste). Il existe une syntaxe lexicale relevant du système collectif de la langue selon laquelle intervient l'acte proprement dit de création. Dans la seconde forme, sémantique, le sujet parlant disposant du matériel lexical de la langue, choisit un terme auquel il confère, selon une motivation d'abord purement personnelle, une signification nouvelle. dans la littérature portant sur le lexique sans bénéficier vraiment d'une définition clairement énoncée retenant les points communs et les points de divergence des deux notions couvertes par les deux termes : néologie et alternance lexicale. Pour Guilbert (1973 : p. 16) radariste est considéré comme un néologisme. Cette illustration est une parfaite manifestation de la confusion dont nous parlions. Notre position diffère de celle de Guilbert et nous arguons que radariste est plutôt un hybride et relève de fait de l'alternance lexicale même si le mot est attesté dans les dictionnaires de la langue française. D'ailleurs, l'étymologie indiquée dans ces mêmes dictionnaires nous renseigne sur son origine anglaise. Il est à noter que néologie et alternance lexicale partagent les mêmes procédés de création, mais se distinguent l'une de l'autre sur plusieurs points :

la néologie s'opère à l'intérieure d'une langue donnée, ce qui n'est pas le cas de l'alternance lexicale qui nécessite la cohabitation d'au moins deux langues. L'exemple donné par Guilbert confirme bien cette assertion :radar + iste = radariste. Nous convenons que radar est un acronyme anglais (Radio Detection And Ranging) et l'affixe (-iste) provient du français ;

la signification de la nouvelle unité lexicale qu'engendre la néologie ne sollicite pas une compétence linguistique complémentaire vu que les deux éléments qui s'adjoignent proviennent de la même langue, contrairement à l'alternance lexicale qui nécessite, dans la plupart des cas, une compétence dans la langue matrice qui sert de base à la création lexicale et aussi une compétence dans la langue qui fournit l'affixe ;

la néologie procède par préfixation et par suffixation tandis que l'alternance lexicale procède généralement par suffixation. Les exemples de préfixation relevés dans l'alternance lexicale ne sont pas pertinents dans les données convoquées et nous n'avons pas jugé utile de s'y attarder ;

la néologie engendre les néologismes et l'alternance lexicale crée des hybrides.

En complément de ces précisions, le tableau suivant expose quelques points de ressemblances et de divergences qui distinguent la néologie de l'alternance lexicale.

Tableau 1 : points de convergences et divergences entre néologie et alternance lexicale

\begin{tabular}{|c|c|c|c|c|}
\hline \multirow{2}{*}{ Néologie } & \multicolumn{4}{|l|}{ Alternance lexicale } \\
\hline & - & + & + & + \\
\hline+ & \begin{tabular}{|lr} 
Néologisme : & \\
recenser, & créer, \\
diffuser & et \\
implanter & les \\
néologismes &
\end{tabular} & $\begin{array}{l}\text { Création d'une nouvelle unité } \\
\text { lexicale }\end{array}$ & $\begin{array}{lr}\text { Création } & \text { par } \\
\text { suffixation : } & \text { le } \\
\text { suffixe } & \text { provient } \\
\text { toujours } & d u \\
\text { français } & \end{array}$ & \\
\hline
\end{tabular}




\begin{tabular}{|l|l|l|l|l|}
\hline- & $\begin{array}{l}\text { Utiliser au moins deux langues } \\
\text { au sein de la même unité } \\
\text { lexicale : r l'hybride } \\
\text { n'appartient ni à la langue } \\
\text { matrice (L1) ni à la langue de } \\
\text { suffixation (L2) }\end{array}$ & $\begin{array}{l}\text { Se réalise en } \\
\text { situation de } \\
\text { contact des langues }\end{array}$ & $\begin{array}{l}\text { Pratiquée } \\
\text { par un sujet } \\
\text { bilingue }\end{array}$ \\
\hline
\end{tabular}

Légende : Distinction néologie et alternance lexicale

12 Les recherches sur les alternances codiques ont-elles traité des alternances lexicales? Cette interrogation trouve sa pertinence dans le contexte qui valide le mécanisme de l'alternance codique : le contact des langues. Les deux mécanismes ont en commun le même contexte d'existence. Si on se situe à ce niveau, on pourrait se demander si l'alternance codique n'englobe pas l'alternance lexicale qui lui serait une manifestation subséquente. Une revue de littérature de quelques travaux traitant de l'alternance codique mettra en lumière les faits abordés et permettra, de ce fait, de situer les différentes positions.

\section{Recherches sur les alternances codiques}

13 A l'heure actuelle, les recherches abordent l'étude des alternances codiques selon deux grands types d'approches. Le premier type est structural: il s'intéresse au fonctionnement linguistique des alternances et cherche à identifier les contraintes systémiques présidant aux alternances (Muysken, 1995; Myers-Scotton, 1993 b). Le second type est, selon Tabouret-Keller (1991), d'ordre social : il s'attache à décrire le fonctionnement discursif des alternances et le rôle joué par ces dernières dans la construction de l'identité des locuteurs qui les produisent. Les travaux s'intéressent ainsi aux contraintes sociales et conversationnelles présidant aux alternances (Auer, 1995 ; Gafaranga, 2001 ; Li, 2002 ; Myers-Scotton, 1993 a).

14 La variété des approches de l'alternance a conduit Lüdi (1991) à insister sur la nécessité d'aboutir à la création d'un modèle général permettant d'intégrer à la fois «des contraintes linguistiques (les propriétés des systèmes linguistiques) à des contraintes psycholinguistiques (les propriétés du cerveau humain) et des contraintes sociopragmatiques (les propriétés sociales et interactionnelles des systèmes sociaux en général et (ou) des systèmes sociaux spécifiques). » Finalement, toutes ces contraintes mises en commun fondent la définition de cet objet de recherches.

15 Certaines des typologies proposées prennent comme point de départ les situations dans lesquelles les alternances apparaissent (Winford, 2003), tandis que d'autres s'appuient sur les caractéristiques des alternances (Auer, 1999). D'autres enfin prennent en compte les compétences des locuteurs dans les différentes langues de l'interaction (Lüdi, 1987). La typologie de Lüdi est, pour sa part, axée sur des microsituations et, plus spécifiquement, sur les interactions où l'on est susceptible d'observer des alternances :

\begin{tabular}{|l|l|l|}
\hline & Bilingue & Monolingue \\
\hline
\end{tabular}




\begin{tabular}{|l|l|l|}
\hline Exolingue & $\begin{array}{l}\text { Interactions entre des locuteurs de } \\
\text { langues différentes }\end{array}$ & $\begin{array}{l}\text { Interactions entre des locuteurs natifs et des } \\
\text { locuteurs non natifs }\end{array}$ \\
\hline Endolingue & Interactions entre bilingues & Interactions entre monolingues \\
\hline
\end{tabular}

16 Auer (1999) propose, quant à lui, de partir des caractéristiques des discours bilingues. Il distingue trois types discursifs où interviennent des alternances :

L'Alternance Conversationnelle (AC) (ou code-switching) pour les cas où la juxtaposition des deux codes est perçue et interprétée comme localement significative par les participants, le choix est marqué et l'alternance est révélatrice en soi ;

Le Mélange de Langues (ML) (ou language mixing), pour les cas où c'est la juxtaposition des deux langues en elle-même qui est significative pour les participants, non pas localement (contextuellement) mais dans le fait même d'employer ce type de discours de manière récurrente ;

La Fusion de Langues (FL) (ou fusedlects) dans des cas de variétés mixtes qui se sont stabilisées, où les locuteurs n'ont plus conscience de la mixité de leurs discours et où ce discours est devenu «langue», comme le michif(Bakker, 1997) ou la media langua(Muysken, 1997). Cela implique une réduction de la variation ainsi que de la régularité des règles morphologiques et morphosyntaxiques.

Ces phénomènes sont conçus comme pouvant se situer dans un continuum AC ML FL ou AC ML, ML FL, ce qui permet de décrire parfois les observations comme phases transitoires (Auer, 1999).

17 Les alternances traitées dans ces travaux relèvent de la langue (niveau macro) et confirme le postulat de départ qui est que les recherches sur les alternances codiques ne sont pas descriptives de toutes les dynamiques (socio)linguistiques observables dans les pays convoqués et autres pays ayant connu les mêmes situations. Elles sont restées descriptives des relations qu'entretiennent les systèmes linguistiques entre eux. Nous n'insinuons cependant pas que ce travail traite de façon exhaustive des dynamiques que manifestent ces environnements linguistiques. Il analyse, à un niveau micro, l'alternance qui découle de la structuration des unités lexicales convoquées afin d'en identifier les langues sollicitées et les rôles que joue chacune d'elles.

Les données présentées ci-dessous seront analysées en fonction de la typologie des alternances lexicales établie, dans lesquelles on observe des mélanges des unités lexicales provenant de différentes langues. Nous nous intéressons d'abord aux structures des alternances lexicales afin d'expliquer leur fonctionnement linguistique.

\section{Structure des alternances lexicales et diglossie lexicale}

18 Cette section est dédiée à la description des structures des alternances lexicales qui ponctuent la pratique du français dans les pays convoqués. Nous considérons ces hybrides comme du mélange de langues parce que les alternances lexicales qui y sont observées rendent impossible - et surtout non pertinente - l'identification formelle d'une langue. Leur structure présente les caractéristiques d'un hybride au sens de l'emploi simultané d'un répertoire linguistique varié à l'intérieur d'une même unité lexicale. Ce mélange de langues n'entrave point la communication. Il n'est source 
d'aucun problème de compréhension ou de clarification chez les locuteurs des pays convoqués et ayant en commun le français.

19 Au regard de la prégnance des affixes utilisées, nous établissons la typologie suivante des alternances lexicales identifiées : celles qui sont à base de langues nationales de type langue nationale + affixe provenant du français. Les hybrides qui en sont issus sont caractéristiques des pays francophones colonisés ayant le français comme langue officielle tels que le Bénin, le Togo, le Burkina-Faso, la Côte-d'Ivoire et le Sénégal. Dans ce cas, les langues nationales fournissent l'essentiel des éléments lexicaux tandis que les apports du français sont essentiellement affixaux. Cette tendance s'inscrit dans le sens de l'hypothèse de Halmari (1997) selon qui la langue matrice est celle qui a la morphologie la plus complexe. La stratégie affixationnelle serait donc celle de prédilection dans des cas comme ceux-ci. Cette explication n'est pas en contradiction avec les critères sociolinguistiques dans le choix d'une langue matrice par le locuteur. On pourrait établir une typologie interne en prenant pour références la catégorie grammaticale des hybrides : les hybrides verbaux et les hybrides nominaux ;

\begin{tabular}{|c|c|c|c|}
\hline$(1$ & \multicolumn{3}{|l|}{ Bénin } \\
\hline & Yayi - Boni + iser & $>$ & yayiboniser \\
\hline & gbotièmi + iser & $>$ & gbotèmiser \\
\hline & \multicolumn{3}{|l|}{ Burkina-Faso } \\
\hline & Sankara + iser & $>$ & sankariser \\
\hline & Compaoré + iser & $>$ & compaoriser \\
\hline & \multicolumn{3}{|l|}{ Sénégal } \\
\hline & mandingue + iser & $>$ & mandinguiser \\
\hline & toubab + iser & $>$ & toubabiser \\
\hline & \multicolumn{3}{|l|}{ Algérie } \\
\hline & Boutéflika + er & $>$ & boutéfliker \\
\hline & gabr +er & > & gabrer (capter des yeux) \\
\hline
\end{tabular}

On y voit apparaitre la création verbale avec le recours de préférence aux suffixes verbaux -er et -ser avec une variante en -iser. Cette variante révèle l'insertion d'une voyelle épenthétique (i) dans un souci conscient ou inconscient d'harmonie. C'est un mécanisme qui permet d'apprécier des satires et des brins d'humour des variantes expressives. Dans le cas de compaoriser, il est créé par les activistes burundais qui, s'inspirant de la chute de Compaoré au Burkina Faso, se mobilisent pour réitérer la même expérience dans leur pays. C'est ce qu'il faut comprendre du titre du blog qui indique : «Burundi : comment on s'apprête à compaoriserNkurunziza »1. Ces hybrides 
manifestent les dynamiques sociales et politiques des pays convoqués et ces verbes en sont des témoins privilégiés.

21 La suffixation verbale est moins productive que la suffixation nominale qui offre plus de possibilités combinatoires et un éventail fourni d'affixes suffixaux. Les données ciaprès exemplifient bien ce constat.

\begin{tabular}{|c|c|c|c|c|}
\hline (2) & Bénin & & & \\
\hline & & Yayi + isme & $>$ & yayisme \\
\hline & & Kérékou + isme & $>$ & kérékouisme \\
\hline & & Soglo + isme & $>$ & sogloisme \\
\hline & & Yayi + iste & $>$ & yayiste \\
\hline & & Kérékou + iste & $>$ & kérékouiste \\
\hline & & Soglo + iste & $>$ & sogloiste \\
\hline & \multicolumn{4}{|c|}{ Burkina-Faso } \\
\hline & & Sankara + isme & $>$ & sankarisme \\
\hline & & Compaoré + isme & $>$ & compaorisme \\
\hline & & Compaoré + iste & $>$ & compaoriste \\
\hline & & Sankara + iste & $>$ & sankariste \\
\hline & \multicolumn{4}{|c|}{ Sénégal } \\
\hline & & Senghor+ isme & $>$ & senghorisme \\
\hline & & Wade + isme & $>$ & wadisme \\
\hline & & Tanor+ isme & $>$ & tanorisme \\
\hline & & Sopi + isme & $>$ & sopisme \\
\hline & & Diouf + isme & $>$ & dioufisme \\
\hline & \multicolumn{4}{|c|}{ Côte d'Ivoire } \\
\hline & & Gbagbo + isme & $>$ & gbagboisme \\
\hline & & Watara + isme & $>$ & watarisme \\
\hline & & Gbagbo + iste & $>$ & gbagboiste \\
\hline & & Watara + iste & $>$ & watariste \\
\hline
\end{tabular}




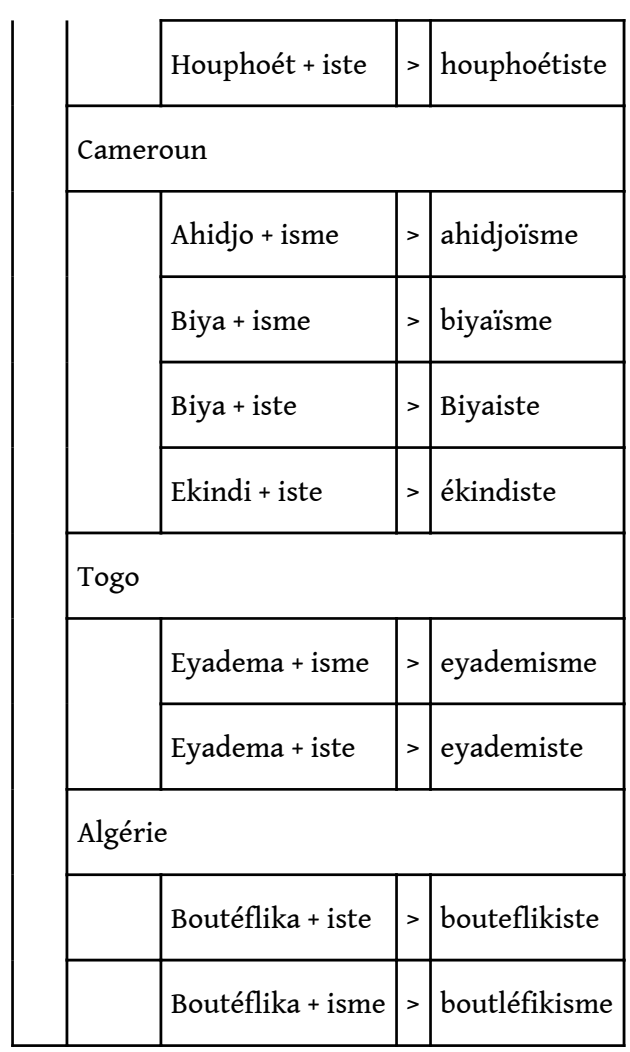

Ces hybrides sont fondés sur la recherche de l'expressivité pour traduire des pensées diachroniques et surtout synchroniques ou des modes de penser. Ils ont une résonance de la satire politique qui dénonce les dérives politiques autoritaires et parfois abusives des politiques africains. C'est aussi l'expression d'une participation active de la population dans la gestion de leur patrie commune face à des dirigeants très peu soucieux de la bonne gestion des deniers publics et aussi très peu préoccupés des souffrances de leur peuple. Bref, c'est une pratique idéologique à laquelle n'adhèrent pas toujours ces politiques africains qui n'y voient pas un acte de liberté d'expression et une manifestation patente de la démocratie mais un acte d'insubordination et d'opposition à leur vision politique.

Pour le cas de la Côte d'Ivoire, nous avons identifié deux cas de dérivation parasynthétique faisant état d'un usage simultané des affixes pro- et -isme. Ces exemples sont très peu productifs et rarement usités dans les autres pays.

\begin{tabular}{|c|c|c|c|}
\hline \multirow[t]{2}{*}{ (3) } & \multicolumn{3}{|l|}{ Côte d'Ivoire } \\
\hline & Pro + Gbagbo + imse & $>$ & progbagboisme \\
\hline & Pro + Watara + imse & $>$ & prowatarisme \\
\hline
\end{tabular}

24 Celles qui sont à base anglaise de type anglais (pidgin english) + affixe provenant du français et qui sont caractéristiques des pays ayant subi une double colonisation, française et anglaise. Ces pays ont généralement deux langues officielles : le français et l'anglais comme c'est le cas du Cameroun ${ }^{2}$; 


\begin{tabular}{|c|c|c|c|}
\hline \multirow[t]{2}{*}{ (4) } & \multicolumn{3}{|l|}{ Cameroun } \\
\hline & Ngrimbah + ter & $>$ & ngrimbahter \\
\hline & Ngrimbah + tique & $>$ & ngrimbahtique \\
\hline & Ngrimbah + tiquement & $>$ & ngrimbahtiquement \\
\hline & Kongossa + eur & $>$ & kongosseur \\
\hline & Call-box + eur & $>$ & call-boxeur \\
\hline & White +iser & $>$ & whitiser \\
\hline & White + isation & $>$ & whitisation \\
\hline
\end{tabular}

La structure des exemples sus-présentés révèle une forte utilisation du pidgin english camerounais. Cela traduit la dynamique du pidgin dans la pratique langagière des Camerounais. Cela se justifie en ce sens qu'elle est une variante libérée de toute contrainte normative qui limite l'expression de la créativité et sur laquelle le locuteur n'a qu'un rôle accessoire, restreint à l'usage de ce qui est et établi. L'anglais normatif se contente de quelques rares exemples (call-box, white).Ce contraste qui transparait de l'usage de l'anglais normatif et de celui du pidgin camerounais dans la création des hybrides, élucide clairement le postulat que la norme handicape le désir créatif des locuteurs et limite le dynamisme linguistique.

Dans les deux cas, le français n'est jamais la langue matrice à laquelle s'adjoignent les affixes. Ce procédé est l'expression d'une certaine maittrise de la langue française ou anglaise par ces locuteurs. L'affixation (spécifiquement la suffixation) est un procédé très peu productif dans les langues nationales des pays concernés (le Bénin en l'occurrence) et permettent généralement de créer des nominaux jamais des verbaux comme c'est le cas dans les alternances lexicales. L'alternance lexicale intervient comme pour combler un vide morphologique. Dans la plupart des exemples convoqués, on identifie les mêmes procédés dérivationnels qu'en français, par exemple partir d'un nominal pour créer un verbe qui est généralement du premier groupe et rarement un verbe du deuxième ou du troisième groupe qui présentent des structures plus complexes.

La structure des alternances lexicales manifeste une répartition fonctionnelle. Les langues nationales et l'anglais (pidgin) fournissent les bases lexicales et l'affixe provient toujours du français. Le rôle de premier niveau est attribué aux langues nationales et à l'anglais (pidgin). Le sens de l'hybride est construit à partir du sens de la base lexicale. Elles remplissent ainsi la fonction haute laissant la fonction basse au français. Ce constat induit une situation de diglossie lexicale. La diglossie lexicale est une situation dans laquelle la structure de l'unité lexicale est révélatrice de la présence de plusieurs langues et où une langue assure toujours la fonction haute (base lexicale) au détriment d'une autre qui remplit toujours une fonction basse (affixe).

Si le français a toujours occupé une position confortable dans la plupart des pays convoqués, à partir de la diglossie lexicale constatée, il parait logique d'émettre l'hypothèse que la pratique des alternances lexicales exposent, inconsciemment peut 
être, la volonté toujours manifestée des citoyens de ces pays de voir les langues locales occupées une place de choix dans le processus d'enseignement apprentissage et dans l'administration. Cette pratique exprime une opposition inconsciente à l'impérialisme de la langue française dans ces pays et dont le témoin est la pratique des alternances lexicales.

L'analyse linguistique choisie n'est pas descriptive des contextes dans lesquels des alternances lexicales de ce type apparaissent. Pour cela, il convient, comme nous le montrons dans le point suivant, de préciser leurs situations et leurs domaines d'emploi.

\section{Situations et domaines d'emploi}

Les pays convoqués dans l'article présentent des situations favorables à la pratique de l'alternance lexicale. Nous fixons quelques critères sociolinguistiques qui valident la vitalité des alternances lexicales. Nous distinguons fondamentalement :

l'usage régulier des alternances lexicales dans les émissions interactives à caractère politique sur les chaînes de radios et de télévisions, sur les réseaux sociaux et dans les journaux (sur support papier ou numérique) ;

l'existence et le développement de productions littéraires écrites et scientifiques comportant des alternances lexicales. Le titre de l'article de Lüdi (1991), "Les apprenants d'une L2 code-switchent-ils et, si oui, comment?", est illustratif de la présence des alternances lexicales dans les travaux scientifiques. On peut clairement identifier la présence du verbe code-switcher qui tient sa base (code-switch-) de l'anglais et son affixe du français (-er) ;

l'apparition et l'acceptation des alternances lexicales dans les pratiques langagières diverses ;

l'usage du français ou de l'anglais comme langue véhiculaire dans des espaces géographiques conséquents et tendant à s'agrandir ;

l'établissement d'une corrélation entre un statut valorisé (au français ou à l'anglais) et le fait même d'utiliser la langue puisqu'il confère à l'énonciateur plus de sérieux et de considération sociale.

Ces hybrides véhiculent des significations sociolinguistiques notamment l'hégémonie de la langue française dans ces différents pays. Le français y est la langue officielle et les autres langues ne se contentent que du statut de langues nationales. C'est ce que manifestent justement les situations et les domaines d'emploi des alternances lexicales. Ils se révèlent comme une caractéristique essentielle des alternances lexicales. Nous nous intéressons d'une part aux situations de leur emploi et d'autre part à leurs domaines d'emploi.

Tableau 1 : situations d'emploi des alternances lexicales

\begin{tabular}{|l|l|}
\hline Situations & Emploi \\
\hline Discours politiques & + \\
\hline Campagne électorale & + \\
\hline Conversations entre amis et sympathisants de partis politiques & + \\
\hline
\end{tabular}




\begin{tabular}{|l|l|}
\hline Informations, médias (journaux, radios, télévisions) & + \\
\hline Pays multilingues francophones et / ou anglophones & + \\
\hline Pays colonisés & + \\
\hline débats politiques & + \\
\hline Théâtre (humour) & + \\
\hline Littérature & + \\
\hline Musique & + \\
\hline Internet & + \\
\hline Recherches scientifiques & + \\
\hline
\end{tabular}

Légende : situations d'emploi

Ces situations sont descriptives de l'emploi des alternances lexicales mais ne permettent pas d'apprécier le choix des langues contrairement aux domaines qui en exposent les différents contextes et qui élucident la répartition fonctionnelle des langues en contact.

Tableau 2 : Domaines d'emploi des alternances lexicales et répartition fonctionnelle des langues

\begin{tabular}{|c|c|c|c|c|}
\hline \multirow{3}{*}{ Domaines d'emploi } & \multicolumn{4}{|c|}{ Répartition fonctionnelle des langues } \\
\hline & \multicolumn{2}{|c|}{ pays francophones } & \multicolumn{2}{|c|}{$\begin{array}{l}\text { pays francophones et } \\
\text { anglophones }\end{array}$} \\
\hline & $\begin{array}{l}\text { langue } \\
\text { matrice }\end{array}$ & affixes & langues matrices & affixes \\
\hline Débats politiques & LN & $\mathrm{F}$ & $\mathrm{LN} / \mathrm{A}$ & $\mathrm{F}$ \\
\hline Discours politiques & LN & F & LN / A & $\mathrm{F}$ \\
\hline Conversations avec des collègues & LN & $\mathrm{F}$ & LN / A & $\mathrm{F}$ \\
\hline Informations (radios, télévisions) & LN & F & LN / A & $\mathrm{F}$ \\
\hline $\begin{array}{l}\text { Journaux (support papier et support } \\
\text { numérique) }\end{array}$ & LN & $F$ & $\mathrm{LN} / \mathrm{A}$ & $\mathrm{F}$ \\
\hline Internet (réseaux sociaux) & LN & $\mathrm{F}$ & $\mathrm{LN} / \mathrm{A}$ & $\mathrm{F}$ \\
\hline Musique & LN & F & LN / A & $\mathrm{F}$ \\
\hline
\end{tabular}




\begin{tabular}{|l|l|l|l|l|}
\hline $\begin{array}{l}\text { Travaux scientifiques (thèses, articles, } \\
\text { mémoires) }\end{array}$ & LN & F & LN / A & F \\
\hline
\end{tabular}

Légende : domaines d'emploi et répartition des fonctions

Le français apparaît aujourd'hui, en nous fondant sur le tableau 2, dans une dynamique de fragilisation du carcan normatif et d'expression des identités locales manifestées par la répartition fonctionnelle des langues dans la création des hybrides. Leur diversification apparaît désormais surtout liée aux caractéristiques sociales des locuteurs, leurs activités et leurs interactions.

31 Le français actuel, parlé sur les ex-territoires d'expansion et de colonisation mais aussi d'autres langues européennes, notamment l'anglais, connaissent une large gamme d'effets de contacts linguistiques: émergence d'unités lexicales hybrides en l'occurrence. Même si ces hybrides ne sont pas appréciés des puristes de la langue française, elles suscitent néanmoins des interrogations sur les évolutions actuelles et futures du français dans la francophonie en contact. La dynamique semble irréversible au regard des domaines et situations qui facilitent leurs usages.

\section{Discussion en guise de conclusion}

Les alternances lexicales sur le fond du mouvement général de la langue sont la traduction de la lutte permanente entre, d'un part, l'esprit de liberté, la recherche du nouveau qui stimule la créativité des locuteurs et notamment des écrivains accomplissant leur fonction d'inventeurs de mots souvent teintés de couleurs nationales et, d'autre part, l'esprit de tradition, de conservation qui la paralyse au nom de la norme et de l'ordre établi. Elles sont indépendantes de la loi propre au système linguistique qui implique à la fois la mutabilité et l'immutabilité ; elles sont l'aspect idéologique du mouvement. Dans les faits, elles charrient les dynamiques sociales, ne serait-ce qu'en vertu de l'alternance lexicale dénominative.

Le modèle socioculturel implique une certaine définition de l'usage de la langue par rapport à une élite cultivée, dont les contours varient selon les époques, les régimes politiques et les sociétés. Une place éminente est accordée naturellement aux professionnels du verbe (les écrivains, les enseignants, les journalistes et autres hauts cadres), qui occupent les places de choix dans la société. La décision d'acception ou de rejet des alternances lexicales ne se situe pas au niveau de la langue, qui détermine seulement sa création, elle s'impose aux sujets parlants selon leur culture et le modèle socioculturel de la société. Ajoutons à tout ceci que dans les faits, le flot de production verbale déversé par les moyens audio-visuels, les journaux, les réseaux sociaux et le prestige dont elle jouit dans la masse parlante fait plus pour la vitalité et l'adoption de l'alternance lexicale que toutes les décisions d'ordre académique ou de politique linguistique. 


\section{BIBLIOGRAPHIE}

ADJERAN, Moufoutaou, «Le français au Bénin : emprunts, niveaux d'appropriation et typologie fonctionnelle ", dansJean Paul Balga et David Abaikaye (dir(s).), Le français et les langues africaines aux lendemains des indépendances en Afrique francophone : bilan et perspectives,Paris,2017, pp. 73-90.

ADJERAN, Moufoutaou, NDAO, Dame et DIOUF Ngari, « Hybridisme dans la pratique du français au Bénin et au Sénégal : un procédé d'enrichissement lexical en situation de contact des langues ", in Multilinguales 9, Béjaïa, 2018, pp. 227-251.

ANOKHINA, Olga, «La contribution des noms abstraits à la formation des néologismes », dansJ.-F. Sablayrolles(dir.), L'innovation lexicale,Paris, 2003, pp. 437-476.

AUBERT, Françoise, Néologie et Idéologie : Élite et son champ dérivationnel dans la langue française contemporaine, in Studidell'Istituto Linguistico 2, 1979, pp. 3-25.

AUER, Peter, "The pragmatics of code switching a sequential approach", in Milroy et Muysken (dir(s).), One speaker, two languages, cross-disciplinary perspectives on code-switching, Cambridge University Press, 1995, pp. 115-135.

AUER, Peter, "From codeswitching via language mixing to fused lects, toward a dynamic typology of bilingual speech", inInternational Journal of Bilingualism 3 (4), 1999, pp. 309-322.

BAGUE, Jean-Marie, " "L'utilisation de mots étrangers" dans un roman ouest-africain de langue français : Monné, outrages et défis d'Ahmadou Kourouma », in Ambroise Queffelec (dir.), 1998, pp. 33-53.

BEAUD, Stéphane, «L'usage de l'entretien en sciences sociales. Plaidoyer pour l'“entretien ethnographique" ", inPolitix35, 1995, pp. 226-257.

CORREARD N'DIAYE, Genéviève / Ndiaye, Aliou / NgnoéSeck / Cheikh Hamallah Traoré (2006), Les Mots du patrimoine : le Sénégal, Paris, 2016.

FISHMAN, Joshua. A., Sociolinguistique, Paris, Nathan, 1971.

FISHMAN, Joshua A, Societal bilingualism, Stable and transitional, in Fishman Joshua, Sociolinguistics, a brief introduction. Rowley,1972, 73-90.

GAFARANGA, Joseph, "Linguistic identities in talk-in-interaction : Order in bilingual conversation", inJournal of Pragmatics, 33, 2001,pp. 1901-1925.

GUILBERT, Louis, « Théorie du néologisme », inCahiers de l'Association internationale des études françaises, 25, 1973, pp. 9-29.

GUILBERT, Louis (1975), La créativité lexicale, Paris, 1975.

GUMPERZ, J. John etHymes, Dell, Directions in sociolinguistics, New York, 1972.

HALMARI, Helena (1997), Government and codeswitching. Explaining American-Finnish,Philadelphie : John Benjamins.

LI, Wei, 'What do you want me to say ?' On the conversation Analysis approach to bilingual interaction. inLanguage in Society 31, 2002, pp. 159-180.

LÜDI, Georges, « Métaphore et néologismes », inTRANEL1, 1980, pp. 9-30. 
LÜDI, Georges, « Aspects énonciatifs et fonctionnels de la néologie lexicale », in TRANEL 5,1983, pp. 105-130.

LÜDI, Georges, Aspects énonciatifs et fonctionnels de la néologie lexicale. Recherches linguistiques 10, 1984, pp. 165-183.

LÜDI, Georges, Devenir bilingue-parler bilingue, Tübingen, 1984.

LÜDI, Georges, Les apprenants d'une L2 code-switchent-ils et, si oui, comment ? Symposium on code-switching in Bilingual studies, theory significance and perspectives, Barcelona : Strasbourg, 1991, pp. 47-71.

MERLE, Gabriel et al.,Néologie lexicale 2, Paris-7, 1987.

MERLE, Griel et al. , Néologie lexicale 6, Paris-7, 1994.

MUYSKEN, Pieter, "Code-switching and grammatical theory", dans MILROY et MUYSKEN (dir(s).), One speaker, two languages, cross-disciplinary perspectives on code-switching,Cambridge, 1995,pp. 177-198.

MYERS-SCOTTON, Carol, Social motivations for code switching, evidence from Africa, Oxford, 1993a.

MYERS-SCOTTON, Carol, Duelling languages, grammatical structures in code switching, Oxford, 1993b.

NZESSE, Ladislas, Le français au Cameroun : d'une crise sociopolitique à la vitalité de la langue française (1990-2008), inLe Français en Afrique, Nice, 2009.

NZESSE, Ladislas, Inventaire des particularités lexicales du français au Cameroun (1990-2015), Le Français en Afrique, Nice, 2015, ILF-CNRS.

POOE, L. C., Contacts des langues et identité : le rôle de L'innovation lexicale dans le cas du scamto,dans Mathieu L. et al. (dir(s).), Autour des langues et du langage. Grenoble, 2008, pp. 315-322.

SABLAYROLLES, Jean-François, « Néologisme : une typologie des typologies », inCahier du CIEL, 1997, pp. 11-48.

SABLAYROLLES, Jean-François, La néologie en français contemporain, Paris 2008.

WINFORD, Donald, An introduction to contact linguistics, Massachusetts, 2003.

ADCAFRIQUE Over Blog, «Le lumubisme, le sankarisme, gbagboisme : système politique d'éveil de conscience des Africains », disponible sur [http://adcafrique.over-blog.com/2019/11/lelumumbisme-le- sankarisme-le-gbagboisme-systeme-politique-d-eveil-de-conscience- desafricains.html], consulté le lundi 3 juin 2019.

AFRIQUE Magazine, « Burkina : Constats et espérance » disponible sur [http:// www.afriquemagazine.com/burkina-constats-et- esp\%C3\%A9rance, consulté le dimanche 2 juin 2019.

CLAUDUS Ivoire, "C'est quoi le gbagboisme », disponible sur [http://claudus.ivoire-blog.com/ archive/2019/10/09/c-est-quoi-le- gbagboisme1.html], consulté le lundi 3 juin 2019.

DEFINISTAIRE Définistaire, « Boutefliker », disponible sur [http://www.definistaire.org/ dictionnaire-des-mots-qui-n-existent- pas/boutefliker-4140], consulté le lundi $1^{\text {er }}$ juillet 2019.

DIRECTSCOOP WordPress, « Côte d'Ivoire, Chef armée rebelle, nouveau chef d'état-major », disponible sur [https://directscoop.wordpress.com/2019/07/07/cote- d \%E2 \%80 \%99ivoire-chefarmee-rebelle-nouveau-chef-etat-major- ouattara/], consulté le dimanche 7 juillet 2019. 
EMPREINTES Mondo, «Abidjan, le philosophe du pile ou face », disponible sur [http:// empreintes.mondoblog.org/2016/06/26/abidjan-le- philosophe-du-pile-ou-face/], consulté le lundi 3 juin 2019.

IWACU Burundi, « Burundi : compaoriserNkurunziza », disponible sur [http://www.iwacuburundi.org/blogs/rolandrugero/burundi- compaoriser-nkurunziza-bujumbura-mediasfacebook-twitter- whatsapp-revolution-amatora2015/], consulté le mercredi 3 juillet 2019.

LA DEPECHE d'Abidjan, « Côte d'Ivoire, passons aux choses sérieuses », disponible sur [http:// www.ladepechedabidjan.info/Cote-d-Ivoire- passons-aux-choseserieuses_a11394.html], consulté le samedi 8 décembre 2019.

LA DEPECHE d'Abidjan, «La vision-conception gbagboiste de la politique », disponible sur [http://www.ladepechedabidjan.info/La-vision- conception-gbagboiste-de-lapolitique_a7903.html],consulté le lundi 27 mai 2019.

LEMATIN Lematin, « La répantance : une valse pour marionnettes bouteflikistes » disponible sur [http://www.lematindz.net/news/3280- la-repentance-une-valse-pour-marionnettesbouteflikistes.html], consulté le vendredi 28 juin 2019.

LEMATIN Lematin, « Le boutefliksme veut s'installer pour longtemps en Algérie », disponible sur [http://www.lematindz.net/news/2672-le- bouteflikisme-veut-sinstaller-pour-longtemps-enalgerie-2-zerhouni- a-la.html], consulté le lundi 23 mars 2020.

NRGUI Nrgui, « Tout sur la Guinée de nrguicom nouvelle république de Guinée : actualités, informations de Guinée Conakry », disponible sur http://www.nrgui.com/tout-sur-la-guineenrguicom-nouvelle- republique-de-guinee-actualites-informations-guinee-conakry/44politique/2385-a-conakry-le-pouvoir-opte-pour-le-gbagboisme, consulté le lundi 3 juin 2019.

WIKTIONARY Wiktionary, « Boutéfliker », disponible sur [https://fr.wiktionary.org/wiki/ boutefliker], consulté le mercredi 28 juin 2017.

\section{NOTES}

1. 1 http://www.iwacu-burundi.org/blogs/rolandrugero/burundi-compaoriser-nkurunzizabujumbura-medias-facebook-twitter-whatsapp-revolutionamatora2015/, consulté le 28 juillet 2019.

2. 2 « Dès le début de la Première Guerre mondiale, Français, Belges et Britanniques attaquèrent le Congo Belge et le Nigéria, puis cernèrent le Kamerun, en lui imposant un blocus maritime. La ville de Douala tomba en 1914, puis toute la région côtière, l'année suivante ; en 1916, les troupes allemandes abandonnèrent le Kamerun. Les Français et les Britanniques créèrent un « condominum» (qui ne dura que le temps de la guerre) pour administrer le territoire arraché progressivement aux Allemands. Ceux-ci ne laissèrent que peu de traces de leur langues, car ils avaient été trop détestés. » " (Nzesse, 2009 :p.22). 


\section{RÉSUMÉS}

La pratique du français et/ou de l'anglais, laisse apprécier des alternances lexicales confondues, jusque-là, à la néologie. De la distinction néologie et alternance lexicale, il ressort que la néologie n'implique pas une situation particulière de l'environnement linguistique. L'analyse des hybrides facilite la lecture de leurs structures, leurs situations et domaines d'emploi.

The practice of French and / or English allows to appreciate lexical-alternations hitherto confused, neology. From the distinction between neology and lexical alternation, it emerges that the neology does not imply a particular situation in the linguistic environment. The analysis of hybrids facilitates the reading of their structures, situations and fields of use.

\section{INDEX}

Mots-clés : alternance lexicale, affixation, hybrides, contact des langues, sociolinguistique Keywords : Lexical alternation, Affixation, Hybrids, Languages Contacts, sociolinguistics

\section{AUTEUR \\ MOUFOUTAOU ADJERAN}

Université d'Abomey-Calavi, Bénin 